\title{
Flagellate Shiitake Mushroom Reaction With Histologic Features of Acute Generalized Exanthematous Pustulosis
}

Richard J. Browning, MD; Ramin Fathi, MD; Mahsa A. Smith, MD; Theodore Alkousakis, MD

\section{PRACTICE POINTS}

- Ingestion of shiitake mushrooms and bleomycin is associated with flagellate dermatitis.

- Acute generalized exanthematous pustulosis (AGEP) is a rare condition associated with certain drug ingestion.

- Flagellate dermatitis caused by shiitake mushroom ingestion may present with histologic features of AGEP.
Physical examination revealed an afebrile man with medium brown skin tone and diffuse, bright red, erythematous patches on the lower legs, axillae, medial forearms, lateral trunk, lower abdomen, and groin. There were distinct flagellate, linear, red patches on the lower legs (Figure 1). In addition, small clusters of 1 - to 2-mm superficial pustules were present on the right upper medial thigh and left forearm with micropapules grouped in the skin folds.

A shave biopsy specimen from a pustule on the right upper medial thigh revealed spongiotic dermatitis with

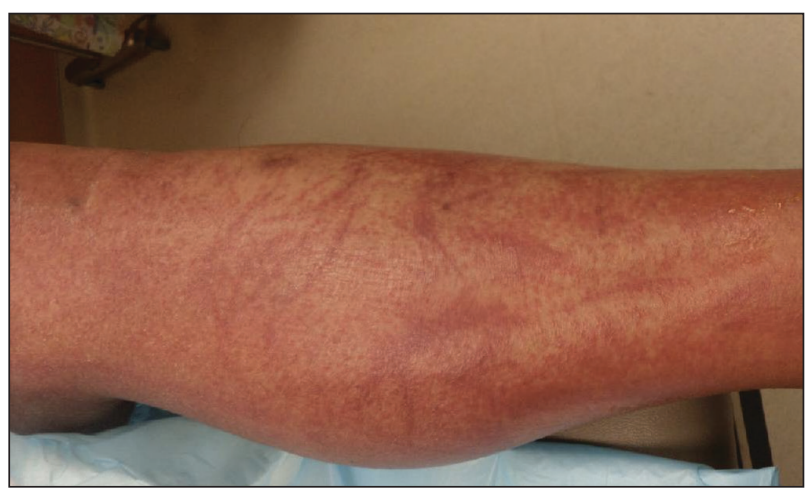

FIGURE 1. Linear, whiplike, red, crisscrossed patches on the lower legs consistent with flagellate dermatitis.

\section{To the Editor:}

A 59-year-old man presented with a severely pruritic rash on the legs, arms, abdomen, groin, and buttocks of 3 days' duration. He reported subjective fever and chills. Prior to the appearance of the rash, the patient and his family had eaten shiitake mushrooms daily for 3 days. He denied any new medications in the last several months or any recent upper respiratory or gastrointestinal tract illnesses. His medical history included type 2 diabetes mellitus and diabetes-induced end-stage renal disease requiring home peritoneal dialysis. His long-term medications for diabetes mellitus, hypertension, benign prostatic hyperplasia, hyperlipidemia, and insomnia included amlodipine, atorvastatin, finasteride, gabapentin, insulin glargine, linagliptin, metoprolol, and mirtazapine.

Dr. Browning is from Presbyterian St. Luke's Medical Center, Denver, Colorado. Drs. Fathi, Smith, and Alkousakis are from the Department of Dermatology, University of Colorado Denver School of Medicine, Aurora. 
neutrophilic subcorneal pustule formation and frequent eosinophils (Figure 2). The dermis contained scattered mixed inflammatory cells including neutrophils, eosinophils, lymphocytes, and histiocytes (Figure 3). These histologic findings were consistent with acute generalized exanthematous pustulosis (AGEP). No biopsy was performed on the flagellate patches due to its clinically distinct presentation and well-established association with shiitake mushroom ingestion.

The patient was treated with triamcinolone ointment and systemic corticosteroids to reduce pruritus and quickly clear the lesions due to his comorbidities. He recovered completely within 1 week and had no evidence of postinflammatory hyperpigmentation from the flagellate dermatitis.

Flagellate dermatitis is an intensely pruritic dermatitis characterized by $1-\mathrm{mm}$, disseminated, erythematous papules in a linear grouped arrangement secondary to koebnerization due to the patient scratching. It was first described in 1977 by Nakamura. ${ }^{1}$ Although it rarely is seen outside of China and Japan, there are wellestablished associations of flagellate dermatitis with bleomycin and shiitake mushroom (Lentinula edodes) ingestion. One key clinical difference between the two causes is that postinflammatory hyperpigmentation changes usually are seen with bleomycin-induced flagellate dermatitis and typically are not present with shiitake mushroom-induced flagellate dermatitis. ${ }^{2}$ Following ingestion of shiitake mushrooms, the median time of onset of presentation typically is 24 hours but ranges from 12 hours to 5 days. Most patients completely recover by 3 weeks, with or without treatment. ${ }^{3}$ Although the pathogenesis of shiitake mushroom-induced flagellate dermatitis is not clear, the most common theory is a toxic reaction to lentinan, a polysaccharide isolated from shiitake mushrooms. However, type I and IV allergic hypersensitivities also have been supported by the time of onset, clearance, severe pruritus, benefit from steroids and antihistamines, and lack of grouped outbreaks in people exposed to shared meals containing shiitake mushrooms. ${ }^{3,4}$ Furthermore, there is a case of patch test-confirmed

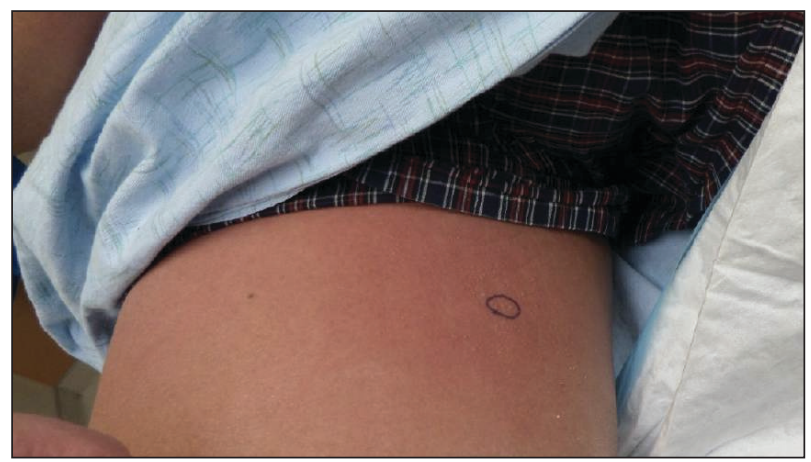

FIGURE 2. Bright red erythematous patch showing the location of biopsied pustule. allergic contact dermatitis to shiitake mushrooms, demonstrating a $1+$ reaction at 96 hours to the cap of a shiitake mushroom but a negative pin-prick test at 20 minutes, suggesting type IV hypersensitivity. ${ }^{5}$ An additional case revealed a positive skin-prick test with formation of a $4-\mathrm{mm}$ wheal and subsequent pruritic papules and vesicles appearing 48 to 72 hours later at the prick site. ${ }^{6}$ Subsequent cases have been reported in association with consumption of raw shiitake mushrooms, but cases have been reported after consumption of fully cooked mushrooms, which does not support a toxin-mediated theory, as cooking the mushroom before consumption likely would denature or change the structure of the suspected toxin. ${ }^{2}$

Acute generalized exanthematous pustulosis is a rare eruption that occurs due to ingestion of a causative agent, usually an antibiotic, and is characterized by the presence of fever and disseminated, erythematous, pinpoint, sterile pustules on the skin and mucous membranes. It affects 1 to 5 persons per million per year, with more than $90 \%$ of cases attributed to drug ingestion. ${ }^{7}$ Spontaneous resolution can be expected within 15 days of its onset; however, there is a mortality rate of up to $5 \%$ that occurs most often in those with severe comorbidities or in older patients, for whom systemic corticosteroid therapy may be justified. ${ }^{7,8}$ A multinational case-control study conducted to evaluate the risk of AGEP associated with certain drugs revealed macrolides (namely pristinamycin); $\beta$-lactam antibiotics including penicillin, aminopenicillin, and cephalosporin; quinolones; hydroxychloroquine; antiinfective sulfonamides; terbinafine; and diltiazem as the most strongly associated culprits. ${ }^{9}$ Our patient's flagellate dermatitis was unique in that it also showed histologic features of AGEP. The pathogenesis of druginduced AGEP has been partially elucidated and involves

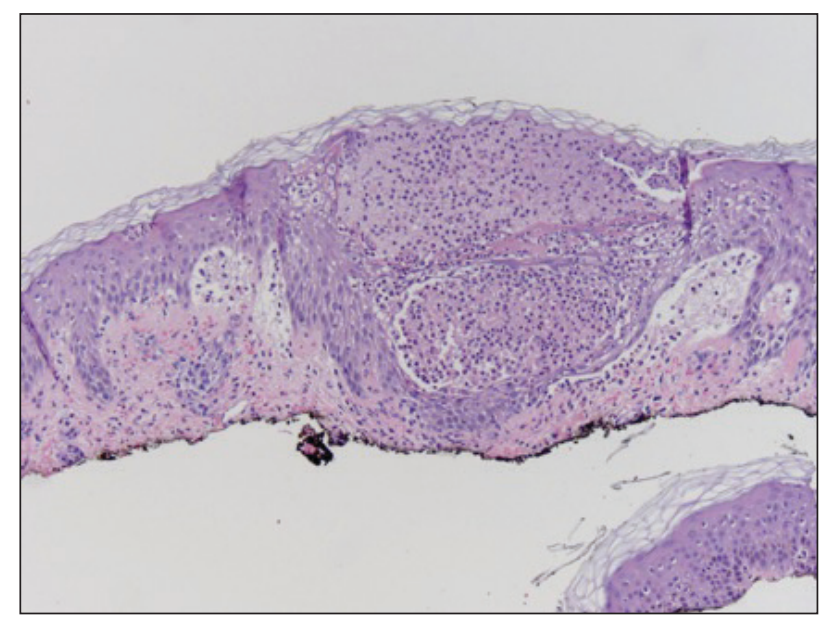

FIGURE 3. A shave biopsy of the right medial thigh showed spongiotic dermatitis with neutrophilic-predominant subcorneal pustule formation and frequent eosinophils. The dermis contained scattered mixed inflammatory cells including neutrophils, eosinophils, lymphocytes, and histiocytes $(H \& E$, original magnification $\times 10)$. 
activation of drug-specific $\mathrm{CD}^{+}$and $\mathrm{CD}^{+} \mathrm{T}$ cells that migrate to the skin and participate in apoptotic signaling of keratinocytes and recruitment of neutrophils and eosinophils, which form subcorneal sterile pustules. ${ }^{7}$ In a study of severe cutaneous adverse drug reactions, $50 \%(7 / 14)$ of patients with AGEP had positive patch tests to the causative agent. ${ }^{10}$ This $\mathrm{T}$ cell-dependent response explains why the condition responds to systemic corticosteroids. Additionally, our case report of shiitake mushroom-induced flagellate dermatitis with histologic features of AGEP suggests that the pathogenesis of flagellate dermatitis may be a T cell-mediated type IV hypersensitivity reaction. The time of onset, lack of grouped outbreaks in those sharing shiitake mushroom-containing meals, severe pruritus, lack of cases demonstrating an anaphylactic or wheal and flare response, benefit of steroids, and a case with histologic features of AGEP all lend support to this theory.

We report a case of shiitake mushroom-induced flagellate dermatitis with histologic features of AGEP. The time course, histologic features of AGEP, absence of new medications, and resolution with discontinuation of shiitake mushrooms lends support of the hypothesis that the pathogenesis of shiitake mushroom-induced flagellate dermatitis is similar to AGEP's type IV hypersensitivity reaction. To further elucidate its pathogenesis, skin prick testing and patch testing with shiitake mushrooms in patients exhibiting shiitake mushroom-induced flagellate dermatitis may prove to be beneficial.

\section{REFERENCES}

1. Nakamura T. Toxicoderma caused by shiitake (Lentinus edodes) [in Japanese]. Jpn J Clin Dermatol. 1977;31:65-68.

2. Chu EY, Anand D, Dawn A, et al. Shiitake dermatitis: a report of 3 cases and review of the literature. Cutis. 2013;91:287-290.

3. Boels D, Landreau A, Bruneau C, et al. Shiitake dermatitis recorded by French Poison Control Centers-new case series with clinical observations. Clin Toxicol (Phila). 2014;52:625-628.

4. Nakamura T. Shiitake (Lentinus edodes) dermatitis. Contact Dermatitis. 1992;27:65-70.

5. Curnow P, Tam M. Contact dermatitis to shiitake mushroom. Australas J Dermatol. 2003;44:155-157.

6. Lippert U, Martin V, Schwertfeger C, et al. Shiitake dermatitis. $\mathrm{Br} \mathrm{J}$ Dermatol. 2003;148:178-179.

7. Fernando SL. Acute generalised exanthematous pustulosis. Australas J Dermatol. 2012;53:87-92.

8. Sidoroff A, Halevy S, Bavinck JN, et al. Acute generalized exanthematous pustulosis (AGEP) - a clinical reaction pattern. J Cutan Pathol. 2001;28:113-119.

9. Sidoroff A, Dunant A, Viboud C, et al. Risk factors for acute generalized exanthematous pustulosis (AGEP)—results of a multinational casecontrol study (EuroSCAR). Br J Dermatol. 2007;157:989-996.

10. Wolkenstein $\mathrm{P}$, Chosidow $\mathrm{O}$, Flechet ML, et al. Patch testing in severe cutaneous adverse drug reactions, including StevensJohnson syndrome and toxic epidermal necrolysis. Contact Dermatitis. 1996:35:234-236 